\begin{tabular}{|c|c|c|c|c|c|}
\hline MUNIBE Antropologia-Arkeologia & $n^{\circ} 67$ & $151-165$ & DONOSTIA & 2016 & ISSN 1132-2217 • eISSN 2172-4555 \\
\hline
\end{tabular}

\title{
Características paleodemográficas de la población recuperada del cementerio de Marialba de la Ribera (Villaturiel, León, España) (S. IV-XIII)
}

\author{
Paleodemographic data from the Marialba de la Ribera cemetery \\ (Villaturiel, León, Spain) (4th-13th centuries AD)
}

PALABRAS CLAVES: estructura por edad y sexo, Demografía, Paleocristiano, Antigüedad Tardía, Edad Media. GAKO-HITZAK: adinaren eta sexuaren araberako egitura, demografia, Paleokristianoa, Antzinatasun Berantiarra, Erdi Aroa KEY WORDS: age-sex structure, Demography, Early Christian, Late Antiquity, Middle Age.

\section{Nieves CANDELAS GONZÁLEZ(1), Álvaro NÚÑEZ CANTALAPIEDRA ${ }^{(1)}$, Josefina RASCÓN PÉREZ ${ }^{(1)}$, Oscar CAMBRA-MOO(1,2), Fernando MUÑOZ VILLAREJO(3), Emilio CAMPOMANES ALVAREDO(3), J. Avelino GUTIÉRREZ GONZÁLEZ ${ }^{(2,4)}$ y Armando GONZÁLEZ MARTíN ${ }^{(1,2)}$}

\section{RESUMEN}

La Paleodemografía es la disciplina que se ocupa del estudio de los parámetros demográficos de las poblaciones pretéritas y su evolución diacrónica, resultando el punto de partida de cualquier investigación posterior. En este trabajo se analizan las características paleodemográficas de los antiguos pobladores de Marialba de la Ribera (Villaturiel, León), a partir del estudio de 269 individuos recuperados en su cementerio. Este yacimiento, con una extensa secuencia cronológica desde época tardorromana a medieval, es considerado de los más importantes del noroeste peninsular. Los resultados del estudio paleodemográfico parecen concordantes con un modelo pretransicional de mortalidad natural, obteniéndose datos afines a los referidos para otras poblaciones de la región con similar cronología. Sin embargo, se ha evidenciado una subrepresentación en el grupo de 0-4 años y una sobrerepresentación en el grupo 5-9 años, que ahora podrían ser explicadas por el uso diferencial del espacio funerario y la excavación parcial del yacimiento.

\section{LABURPENA}

Paleodemografia da iraganeko populazioen parametro demografikoak eta haien bilakaera diakronikoa aztertzeaz arduratzen den diziplina. Ondoren egin beharreko edozein ikerketatarako abiapuntua izaten da. Lan honetan, Marialba de la Riberako (Villaturiel, Leon) garai bateko biztanleen ezaugarri paleodemografikoak aztertu dira bertako hilerrian berreskuratu dituzten 269 banakoren ikerketatik abiatuta. Aztarnategi hori da penintsulako ipar-mendebaldeko garrantzitsuenetako bat eta sekuentzia kronologiko zabala jasotzen du erromatar berantiarretik hasi eta Erdi Arora arte. Ikerketa paleodemografikoaren emaitzak, itxuraz, bat datoz hilkortasun naturalaren trantsizio aurreko ereduarekin eta antzeko kronologia duten eskualdeko beste populazio batzuetarako aipatutakoen antzeko datuak lortu dira. Dena den, 0-4 urte artekoen taldean ordezkaritza txikiegia eta 5-9 urtekoen taldearen gehiegizko ordezkaritza ikusi dira. Ehorzketarako eremuaren eta aztarnategiaren indusketa partzialaren arteko erabilera diferentziala izan daiteke horren guztiaren azalpena.

\begin{abstract}
The Archaeology and Physical Anthropology represent a multidisciplinary approach from which human past researchers could enrich their archaeological sites interpretations. The interaction of both disciplines permit overpass the analysis of isolated individuals in a specific archaeological context, and to reach a holistic understanding of the entire osteological remains recovered. Paleodemography deals with the study of demographic structure in this archaeological populations and their diachronic evolution, and is an essential starting point for any investigation that involve inferences related with the biology and behaviour of past populations.

Taking into account the particular historical context, being an important Early-Christian monument founded in the $4^{\text {th }}$ century and used as a cemetery until the $13^{\text {th }}$ century, and due to the exceptional stage of preservation presented by the skeletal material, Marialba de la Ribera is
\end{abstract}

\footnotetext{
(1) Laboratorio de Poblaciones del Pasado, Departamento de Biología, Facultad de Ciencias, Universidad Autónoma de Madrid. 28049 Madrid. mnieves.candelas@titulado.uam.es alvaro.nunnez@estudiante.uam.es; josefina.rascon@predoc.uam.es; oscar.cambra@uam.es; armando.gonzalez@uam.es

(2) Grupo de Investigación en Arqueología Antigua y Medieval, Universidad de Oviedo. 33011 -Oviedo.

(3) TALACTOR, S.L Arqueología y Patrimonio. fernando@talactor.com; emilio@talactor.com

(4) Departamento de Historia. Facultad de Geografía e Historia. Universidad de Oviedo. Campus de Humanidades. 33071 - Oviedo. avelino@ uniovi.es
} 
one of the most important archaeological location of the NW of the Iberian Peninsula. The archaeological site was firstly excavated in the 60's by Deutsches Archäologisches Institut (DAI) of Madrid, who described the main building as a martyrdom church (martyrium or basilica), and recovered at least 100 skeletons of the inside and the surroundings of this monument. Posterior field works, carried out in 2009 , revealed an important cemetery that finally permitted us to carry out the analysis of 269 individuals that were recovered in the different areas of the site.

This paper presents the first approach of the demographic analysis of the archaeological population recovered at the cemetery of Marialba de la Ribera (Villaturiel, León). Almost all individuals were age characterized and assigned to a concrete age group. The sample was composed by 119 non-adult and 150 adult individuals. Concerning sex determination, it was possible to ascribe 123 adult individuals and using a life table to estimate 25.55 years old as the population life expectancy at birth.

Taking into account our results it seems that the archaeological population recovered at Marialba de la Ribera presents a natural or pretransitional mortality pattern in which the life expectancy at birth reaches a maximum peak in the 10-14 age group and a minimum in the senile adults group. This pattern is similar to the one described in other osteoarchaeological samples recovered in the nearby regions and with similar chronologies. Despite this, it was detected an important bias concerning the individuals from the younger group ages. Those individuals from the 0-4 age group were underrepresented and on the contrary, individuals from 5-9 age group appeared over-represented.

The contrast of all this data with the heterogeneous spatial distribution of the tombs described in the cemetery has permitted us to propose that it exist an intentional use of the funerary space in the different areas of the site. It is probably that those areas still unexcavated, host the individuals of the non-adult age groups underrepresented in the sample. We expect that this preliminary result stimulates future interventions in the archaeological site, which would permit us to corroborate this hypothesis.

\section{1.- INTRODUCCIÓN}

La Arqueología y la Antropología física conforman una plataforma de interacción científica multidisciplinar que, a través de la retroalimentación, configura una aproximación completa a la herencia cultural transmitida. Por tanto, el estudio de alguno de los componentes de forma individualizada y unilateral produciría un sesgo que dificultaría la correcta interpretación de cualquier población arqueológica.

Por un lado, el estudio antropológico de los restos óseos es considerado una parte fundamental en el conocimiento de las poblaciones del pasado, siendo la estructura y dinámica poblacionales piezas clave del mismo. Partiendo del estudio osteológico de los individuos pueden obtenerse diferentes patrones poblacionales, los cuales generarán un perfil demográfico de la población analizada que posteriormente podrá ser utilizado como indicador de la esperanza de vida, la mortalidad y el crecimiento de la población (HOPPA Y VAUPEL, 2002). Por otro, la información arqueológica, derivada del análisis de la estructura y organización funeraria, puede proporcionar información muy útil a partir del estudio de la orientación de las tumbas (BROWN, 1983), distribución y uso de ajuares, que pueden describir a su vez identidades de grupo, familia o, simplemente, una gran variedad de tradiciones funerarias a lo largo del tiempo (RUIZ Y CHAPA, 1990). También se pueden realizar estimaciones del tamaño de la población a partir de diversos factores relacionados, como pueden ser el número total de tumbas, densidad (número de tumbas por superficie), número de individuos por tumba, periodo de utilización y superficie del cementerio (BUIKSTRA, 1976).

A pesar de todas las posibles aportaciones multidisciplinares de esta metodología, este planteamiento no está exento de dificultades. El principal obstáculo que es necesario sobrepasar en las investigaciones sobre restos osteoarqueológicos se relaciona con la pequeña proporción de individuos que habitualmente están disponibles para su análisis. Tal y como lo expresa WAL-
DRON (1994), la población estudiada tras los procesos de enterramiento, preservación y excavación, suele representar solamente una pequeña parte de la población total, de manera que los resultados obtenidos no serán extrapolables a la población biológica de origen. No obstante, a través de un abordaje multidisciplinar y el correcto uso de las diversas metodologías desarrolladas a lo largo de los años en el estudio de la Paleodemografía, es posible obtener resultados fiables que incrementarán el conocimiento de las poblaciones pretéritas. En el presente trabajo se exponen los resultados relativos a la población arqueológica recuperada del cementerio de Marialba de la Ribera (Villaturiel, León).

\section{2.- EL YACIMIENTO DE MARIALBA DE LA RIBERA}

\subsection{Contexto arqueológico}

En 1925, dentro del "Catálogo Monumental de España. Provincia de León (1906-1908)", se hace mención por primera vez al conjunto arqueológico de Marialba de la Ribera. Se realiza una breve reseña a partir de los apuntes y dibujos que Inocencio Arredondo recopiló en sus primeras intervenciones en el yacimiento, que se remontan al año 1890, y cuyos resultados nunca aparecieron publicados. En esta reseña se describe el conjunto arqueológico como un "antiquísimo santuario cristiano" y se menciona un antiguo documento del año 1095 que lo nombra como "Sancta María de Alva" (GÓMEZ-MORENO, 1925). En la actualidad, la basílica paleocristiana de Marialba de la Ribera está considerada de las más importantes del noroeste de la Península Ibérica, habiendo sido declarada bien de interés cultural como zona arqueológica en el año 2000 (B.O.E. núm. 178, de 26 de julio de 2000).

Territorialmente, el yacimiento arqueológico de Marialba de la Ribera, se localiza en el municipio de Vi\|laturiel, a siete kilómetros al sur de la ciudad de León. En las cercanías existe una antigua vía de comunicación entre Legio (León) y Lancia, ciudad astur-romana, que mantuvo su uso a lo largo de toda la Edad Media. 
El yacimiento también se encuentra próximo a ad Legionem (Puente Castro), cuyos territorios tuvieron un papel fundamental en la introducción y expansión del cristianismo en la Península Ibérica y, por tanto, en la transformación de las sociedades tardoantiguas (GUTIÉRREZ Y BENÉITEZ, 1996).

El edificio principal de Marialba, de planta basilical con cabecera ultrasemicircular, fue construido en el siglo IV, con una orientación norte-sur (impropia de las basílicas cristianas); en realidad sería el principal de un conjunto arquitectónico mayor, del cual aún desconocemos su extensión real y su finalidad; quizás podía tratarse de una villa vinculada a Legio, de la cual el edificio basilical sería el aula o espacio de representación principal, pero sin el carácter de basílica martirial que se le ha atribuido (GUTIÉRREZ et al., 2010; GUTIÉRREZ, 2015). Su conversión en lugar de culto se produciría, en realidad, en fases posteriores; a partir del siglo $V$ se transformó progresivamente en un espacio funerario y cultual mediante la adición diacrónica de varios elementos, como una cabecera triconque inscrita en la inicial ultrasemicircular y una serie de trece tumbas inscritas en ella, lo que parece constituir un espacio funerario a modo de mausoleo o martyrium focalizado en la cabecera. Las reformas prosiguieron con la construcción sucesiva de cuatro grandes apoyos de bóveda en los ángulos de la nave, un banco perimetral exterior y un nártex o pórtico funerario a los pies, lo que indica la extensión del uso funerario al resto del edificio, convertido entonces en una basílica funeraria. Ya en época visigoda, en los siglos VI-VII, se añadió un baptisterio al exterior noroeste, formado por una pila oval con escalones afrontados y varias estancias en torno suyo, que irían ornamentadas ricamente con mosaicos, vidrios, placas de mármol y otros elementos arquitectónicos como bases, fustes y capiteles, hallados reutilizados en diversas tumbas posteriores. Esta incorporación indica claramente el uso como iglesia bautismal o parroquial, para la administración sacramental a una amplia comunidad del entorno. De hecho, en esta época continúan los enterramientos, en el pórtico o nártex y en torno al baptisterio, acompañados en ocasiones de las ofrendas y objetos características de época visigoda: botellas o diotae funerarias, armas (lanzas, cuchillos o puñales), broches de cinturón o anillos (GUTIÉRREZ, 2015).

En el siglo VIII el edificio es progresivamente desmantelado y reutilizado como un asentamiento campesino. El uso religioso y funerario se recuperaría posteriormente, a partir del siglo $\mathrm{X}$, extendiéndose por el interior y exterior del edificio durante varios siglos, al menos hasta el siglo XII ó XIII, a juzgar por dataciones radiocarbónicas y los objetos que acompañan algunos enterramientos (GUTIÉRREZ et al., 2010; GUTIÉRREZ, 2015). Cabe destacar que en el exterior occidental de la cabecera (área 1000 sector 1, vid. Figura 1) se documentó arqueológicamente toda la secuencia funeraria completa, desde el siglo IV al siglo XIII, superpuesta en cinco niveles de enterramientos, de los cuales el superior está compuesto por una agrupación de enterramientos infantiles.

Gran parte de la importancia del estudio arqueológico y antropológico dentro del yacimiento de Marialba de la Ribera radica en el amplio espectro temporal que cubre su uso y ocupación. Esto permite plantear y llevar a cabo una gran diversidad de estudios comparativos, en los que estarán representados tanto individuos de época tardorromana, como visigoda y medieval, si bien es cierto que la mayoría de los individuos hallados en el cementerio pertenecen a épocas alto y plenomedievales.

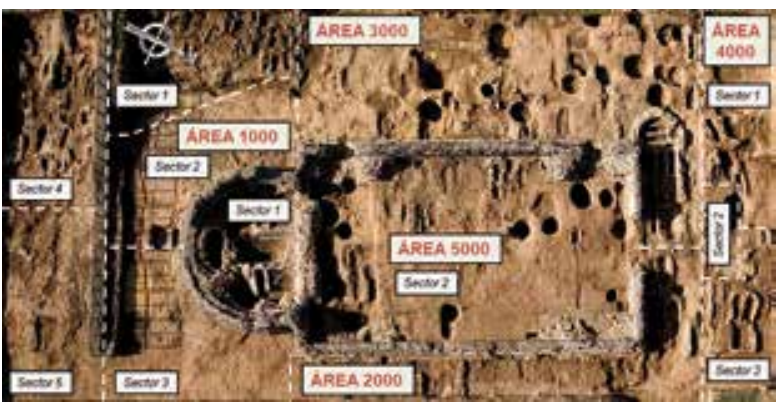

Fig. 1. Vista aérea del yacimiento de Marialba de la Ribera (Villaturiel, León) que muestra las áreas y sectores excavados. / Aerial view of the excavated area in Marialba de la Ribera archaeological site (Villaturiel, León, Spain).

\subsection{Cronología de las intervenciones en el ya- cimiento}

Las intervenciones arqueológicas en el yacimiento de Marialba de la Ribera se realizaron en dos épocas bien diferenciadas. En 1967 se inició la primera fase de la excavación llevada a cabo por parte del Instituto Arqueológico Alemán, liderada por su director, Helmut Schlunk, y Theodor Hauschild, en colaboración con el Instituto Leonés de Estudios Romano-Visigóticos. En esta primera fase, que abarca hasta 1969, se descubrieron los restos óseos situados en el interior, y en las zonas próximas al nártex y baptisterio. El número de tumbas recuperadas en esta campaña alcanza la centena, atendiendo a la descripción ofrecida por HAUSCHILD (1968). Sólo una pequeña parte de estos restos óseos exhumados fueron estudiados por CARRO (1970), centrando su investigación en 18 cráneos de individuos adultos que, mayoritariamente, habían sido recuperados en el interior de la nave.

Cuarenta años más tarde, en 2009, dio comienzo la segunda fase de la excavación promovida y financiada por la Fundación de Patrimonio Histórico de Castilla y León, bajo la dirección científica del Prof. Dr. José Avelino Gutiérrez González, en la que se excavan los restos óseos de 237 tumbas. Durante esta segunda fase se llevó a cabo una sectorización del yacimiento, organizándose en cinco áreas (1000, 2000, 3000, 4000 y 5000 ) subdivididas a su vez en sectores (Figura 1), algunos previamente estudiados y otros de nueva 
excavación. La participación de antropólogos en los trabajos de campo en esta segunda intervención favoreció la exhaustiva recuperación de los restos y la documentación precisa de la información relevante de cada enterramiento.

Actualmente la superficie estudiada del yacimiento arqueológico se aproxima a los $2000 \mathrm{~m}^{2}$, aunque se considera que su extensión real puede ser mucho mayor. En el año 2011 se llevó a cabo una exploración con georradar de $8000 \mathrm{~m}^{2}$ cuyos resultados permiten intuir la existencia de grandes construcciones al norte de la edificación, interpretadas como el posible hábitat romano que dio origen al conjunto arqueológico que se conoce en la actualidad. Este hecho iría en consonancia con la situación geográfica estratégica previamente mencionada.

El principal objetivo de la segunda intervención arqueológica consistió en la documentación arqueológica del monumento basilical y su entorno inmediato. Los restos óseos humanos ahora estudiados han permitido caracterizar la estructura de la población arqueológica en función de las variables biológicas edad y sexo, valorando la distribución de los mismos a lo largo del espacio funerario. Partiendo de dicha caracterización, el estudio paleodemográfico se desarrolla a fin de conocer los detalles relativos a la esperanza de vida y mortalidad en las distintas etapas del ciclo vital y, finalmente, obtener datos que permitan establecer comparaciones en términos de estructura y dinámica poblacional con yacimientos próximos en espacio y época. Los resultados que se ofrecen en el presente trabajo responden a la primera etapa del estudio, en el que se incluye también el análisis diacrónico de la distribución de individuos en las diferentes fases, además de otros aspectos (paleodieta, paleopatología, marcadores de actividad, etc.).

\section{3.- MATERIAL Y MÉTODOS}

\subsection{Muestra de estudio}

Los restos óseos humanos analizados en la investigación proceden de las 237 tumbas excavadas durante la campaña de 2009, cuya muestra queda constituida por un número mínimo de individuos estimado en 269. La preparación del material osteoarqueológico, para poder llevar a cabo su estudio en condiciones óptimas, incluyó la limpieza y reconstrucción de los restos óseos, tras lo que se procedió a la identificación e inventario de todos los elementos de cada esqueleto mediante una base de datos diseñada ad hoc.

\subsection{Caracterización antropológica individual: preservación, edad y sexo}

En primera instancia, se evaluó el grado de preservación de cada individuo a través de la metodología propuesta por RASCÓN et al. (2011). Esta metodología se basa en la determinación, a partir de la observación macroscópica de los restos óseos, de una variable denominada Estado de Alteración Tafonómica (EAT). El EAT describe conjuntamente la completitud del esqueleto (número de unidades anatómicas preservadas) y la calidad del hueso, determinada por la degradación macroscópica observable a simple vista en los restos preservados. Seguidamente, los datos relativos a la edad y sexo de los individuos se obtuvieron aplicando los métodos más apropiados, atendiendo a las diferencias halladas en las distintas etapas del ciclo vital. En el caso de los individuos no-adultos, la estimación de la edad de muerte se realizó, en primera instancia, a partir del estándar de erupción y calcificación dental propuesto por UBELAKER (1978). El rango de incertidumbre que proporciona este método cualitativo aumenta con la edad; para paliar este efecto en la medida de lo posible, se complementó la estimación de la edad con la utilización de los métodos cuantitativos propuestos por LIVERSIDGE et al. (1993), para individuos hasta 5,4 años de edad, y por DEUTSCH et al. (1985) para el primer año de vida. Además se utilizó el método propuesto por IRURITA et al. (2014), que contempla individuos entre los 5 meses de gestación y los 6 años, destacado por tratarse de la única metodología desarrollada sobre una colección de referencia peninsular. Todos los métodos citados se basan en el cálculo de ecuaciones de regresión que relacionan la longitud de los gérmenes dentales en calcificación con la edad cronológica de los individuos de las colecciones documentadas sobre las que se desarrollaron. Las ecuaciones calculadas son lineales $(y=a x+b)$ para los dientes de leche y cuadráticas $\left(y=a+b x+c x^{2}\right)$ para los permanentes, salvo en el caso del método de IRURITA et al. (2014) quienes describen un mejor ajuste para la dentición de leche a partir de ecuaciones exponenciales $\left(y=a x \cdot e^{b x}\right)$.

Mediante una hoja de cálculo se compararon los resultados individuales obtenidos por cada método, tanto numérica como gráficamente. La asignación de la edad de muerte para cada individuo se realizó, de este modo, en función de las diferencias encontradas entre media y mediana por cada método, así como del rango de dispersión de los resultados obtenidos. En cada asignación se priorizaron aquellos métodos que resultaban más apropiados considerando las diferentes etapas del ciclo vital (Figura 2). Como metodología complementaria en la estimación de la edad de los individuos no-adultos, especialmente en aquellos casos en los que no fue posible la utilización de la dentición debido a la ausencia de piezas dentales o al deficiente estado de preservación de las mismas, se utilizaron los métodos compendiados en FAZEKAS Y KÓSA (1978) y SCHEUER Y BLACK (2000). Estas metodologías permiten estimar la edad a partir de las dimensiones de diferentes huesos del esqueleto postcraneal, entre ellos algunos de los más representados y mejor preservados de la colección (fémures, tibias, húmeros, cúbitos, radios, clavículas y 
escápulas). Finalmente, los individuos no-adultos cuya edad de muerte pudo ser estimada en cohortes anuales, fueron asignados a un grupo de cinco años de amplitud (0-4, 5-9, 10-14, 15-19 años) para favorecer la homogeneidad en los análisis. De igual modo, los individuos adultos fueron divididos en otros cuatro grupos denominados "adultos jóvenes", "adultos", "adultos maduros" y "adultos seniles", siguiendo la propuesta basada en el patrón de desgaste dental de MILES (1963). En cuanto a los individuos cuya edad no pudo ser precisada, se distribuyeron proporcionalmente a lo largo de los grupos establecidos, de manera similar a lo expuesto por WALDRON (1994) y GONZÁLEZ (1999).

La determinación del sexo se llevó a cabo únicamente sobre los adultos, debido a la dificultad inherente al estudio de los caracteres sexuales en individuos con edades de muerte estimadas inferiores a la maduración sexual, ya que no es posible analizar características que aun no se han manifestado en el esqueleto (LEWIS, 2007; GONZÁLEZ, 2008). La única excepción es un individuo del grupo 15-19 años que presentó caracteres diagnósticos, motivo por el cual fue posible su inclusión en un grupo concreto. Se priorizaron aquellos métodos que atienden a las características morfológicas y métricas de la pelvis por ser la región anatómica que permite esta identificación con mayor precisión, debido al papel fundamental que tiene en la reproducción (FEREMBACH et al., 1980; BRUZEK Y MURAIL, 2006). Como metodología principal se utilizó la herramienta estadística conocida como Diagnose sexuelle probabiliste o DSP (MURAIL et al., 2005), que compara los resultados obtenidos de la combinación de entre cuatro y diez medidas del hueso coxal del individuo con datos de colecciones documentadas de cuatro continentes, y cuya capacidad de discriminación ha resultado superior al 90\% (SÁNCHEZ-MEJORADA et al., 2011). Además, y de especial utilidad en aquellos casos en que el estado de preservación de esta región anatómica no permitió aplicar la metodología cuantitativa anteriormente descrita, se empleó un amplio abanico de métodos

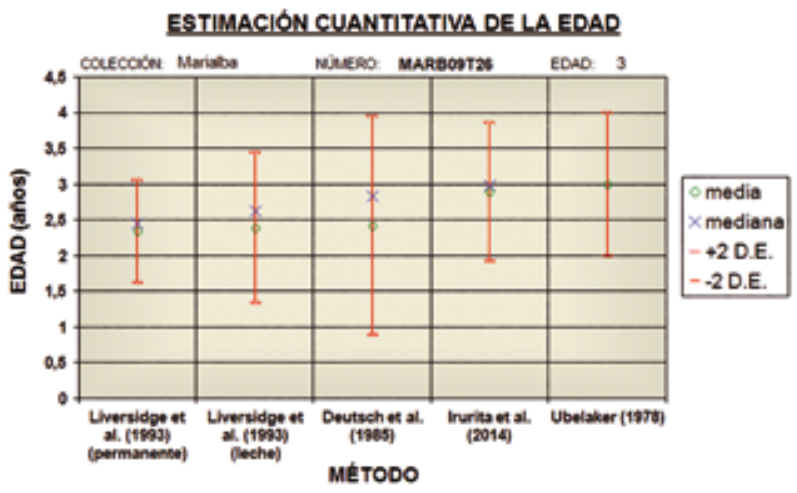

Fig. 2. Ejemplo de la estimación de la edad de muerte del individuo T-26. Los resultados obtenidos por cada método se comparan numérica y gráficamente. / T-26 individual age estimation using the contrast of different graphical and numerical methodologies. morfológicos de uso tradicional en dicha determinación (PHENICE, 1969; FEREMBACH et al., 1980; BRUZEK et al., 1996; BRUZEK, 2002), observándose el grado de coherencia y discrepancia de los resultados obtenidos por cada uno de ellos para paliar, en la medida de lo posible, la subjetividad y el posible error intra-observador a la hora de valorar cada característica estudiada. Finalmente, en aquellos casos en los que los coxales no presentaron el grado de preservación necesario o se encontraron ausentes, se recurrió a los métodos morfológicos propuestos por FEREMBACH et al. (1980) para cráneo y mandíbula.

\subsection{Metodologías aplicadas en el estudio pa- leodemográfico}

Se utilizaron las diferentes aproximaciones que se detallan a continuación, a fin de obtener enfoques lo suficientemente diversos para generar un conocimiento apropiado de la estructura demográfica del conjunto estudiado.

En primer lugar se calculó la tabla de vida, probablemente la forma más comúnmente empleada para el estudio de la mortalidad en el pasado, cuyo desarrollo fue propuesto por ÁCSADI Y NEMESKÉRI (1970). Se trata de una aproximación estática, que no permite observar patrones temporales ni cambios diacrónicos. Son modelos que representan la variación a través de las diferentes etapas del ciclo vital humano, representadas en intervalos, de determinados parámetros poblacionales tales como la esperanza de vida, frecuencia relativa de muertos, porcentaje de supervivientes, probabilidad de muerte (cociente de mortalidad), número de años vividos y número de años por vivir.

A fin de valorar el comportamiento de los individuos no-adultos, la segunda aproximación se basó en la comparación del perfil de mortalidad obtenido con las tablas-tipo desarrolladas por LEDERMANN (1969), establecidas a partir de datos de demografía histórica, y que muestran el intervalo de confianza del cociente de mortalidad en el que debería situarse una población que presente una esperanza de vida al nacimiento determinada. Mediante la comparación con los datos correspondientes a valores de este parámetro comprendidos entre los 25 y 35 años, intervalo en el que se supone deberían encontrarse la mayor parte de las poblaciones prehistóricas e históricas, se pretende contrastar si el perfil de mortalidad obtenido, expresado como el valor del cociente de mortalidad a lo largo de los grupos de edad, se ajusta al de una población sometida a mortalidad natural, sin sesgos que alteren la estructura por edades que cabría esperar en ese supuesto.

La tercera metodología empleada fue la utilización de los estimadores paleodemográficos. Propuestos por MASSET Y BOCQUET (1977), constituyen una herramienta para eliminar el posible sesgo ocasionado, por un lado, por la falta de precisión en la estimación de la edad de muerte de los adultos, clasificándolos den- 
tro de una única categoría y, por otro, por la frecuente subrepresentación de los individuos entre 0 y 4 años, que se ha atribuido tradicionalmente a la preservación diferencial de los mismos. Los parámetros (p. ej., esperanza de vida al nacimiento y cociente de mortalidad) se calculan de este modo a partir del Índice de Juventud (I.J.), que es la proporción entre el número de individuos entre 5 y 14 años y el número total de individuos adultos de la población, los mayores de 20 años.

\section{RESULTADOS}

\subsection{Descripción general de la población ar- queológica}

El cementerio de Marialba de la Ribera se caracterizó por una baja tasa de reutilización del espacio funerario, debido a que los restos óseos recuperados, un total de 269 individuos hallados en 237 tumbas, determinaron una proporción de 1,14 individuos/enterramiento. Al evaluar el Estado de Alteración Tafonómica (EAT) de los individuos, el 59\% fueron clasificados como bien preservados, el $28 \%$ se incluyeron en la categoría de preservación intermedia y apenas un 13\% fueron categorizados como mal preservados, por lo que la colección en su conjunto pudo clasificarse como muy bien preservada macroscópicamente.

La muestra de 269 individuos resultó estar compuesta por 119 no-adultos y 150 adultos. Así, los no-adultos representaron el $44 \%$ de la población arqueológica, mientras que los adultos constituyeron el $56 \%$. Las metodologías aplicadas en la determinación de la edad de muerte permitieron organizar en cohortes anuales al 92\% de los individuos no-adultos (109 de 119). Igualmente, los individuos adultos pudieron ser distribuidos en los distintos grupos de edad en un porcentaje del 73\% (109 de 150). De este modo tan sólo 10 individuos no-adultos y 41 adultos, el 19\% de la muestra total, fueron atribuidos a un grupo $u$ otro atendiendo a la distribución proporcional de los mismos.

En la Figura 3 se puede observar la distribución resultante por grupos de edad del total de individuos recuperados en el yacimiento de Marialba de la Ribera en la campaña de excavación del año 2009. Los grupos más representados coincidieron con la primera y segunda categoría de edad (0-4 y 5-9 años), así como con los adultos y adultos maduros, es decir, las primeras y últimas etapas del ciclo vital. De igual modo, las menores frecuencias se situaron en las etapas intermedias, concretamente en los individuos no-adultos de entre 10 y 19 años y en los adultos jóvenes. Los adultos seniles mostraron una frecuencia cercana a la máxima hallada en etapas intermedias, similar a los adultos jóvenes.

En cuanto a la distribución por sexos, fue posible obtener resultados concluyentes en 123 de los 150 adultos recuperados (82\%) y en un individuo perteneciente a la categoría de 15-19 años, quedando asignado a la categoría "alofisos" un adulto joven que presen- tó igual número de caracteres de un sexo y otro. El resto fueron considerados como indeterminables, debido a la deficiente preservación presentada en las regiones diagnósticas o por la ausencia de las mismas. Se clasificaron 61 individuos como masculinos y 63 como femeninos, por lo que se obtuvo una distribución cercana al $50 \%$ que, además, se mantuvo suficientemente estable a lo largo de los distintos grupos etarios (Figura 4).

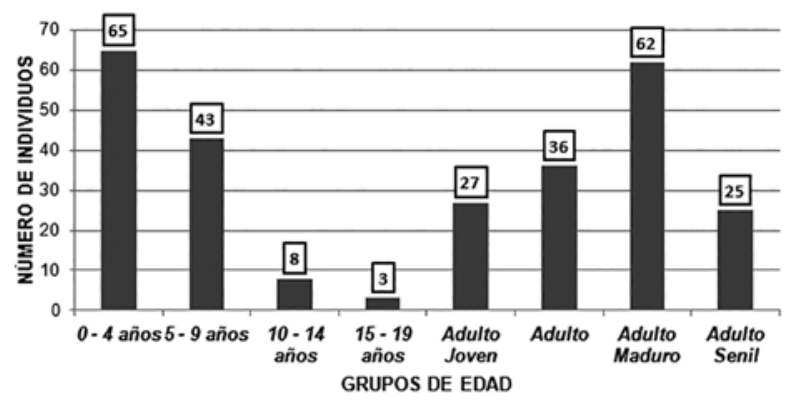

Fig. 3. Distribución por grupos de edad de la totalidad de los individuos recuperados ( $\mathrm{N}=269)$. / Age group distribution of the 269 individuals recovered.

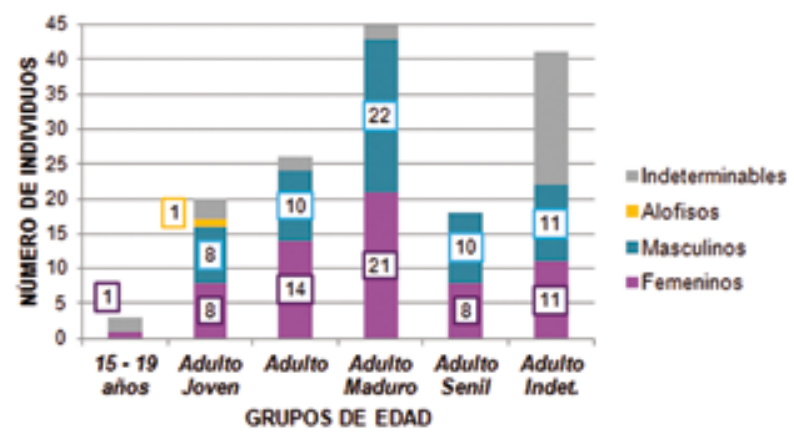

Fig. 4. Distribución por sexos dentro de cada categoría de edad de los individuos recuperados. / Age groups distribution by sex.

Al analizar la distribución de individuos atendiendo a la organización del yacimiento por áreas y sectores, fue posible observar una distribución heterogénea de los enterramientos en el espacio funerario (Figuras 5 y 6). El Área 1000, situada en torno al ábside del monumento y que comprende también la zona sur de la intervención, presentó el mayor número de individuos recuperados en la campaña del 2009 con un total de 191 esqueletos, siendo el Sector 1 el que obtuvo mayor representación $(\mathrm{N}=112)$. Igualmente, se puede destacar que tanto en el Sector 3 del Área 1000 como en el Área 5000 en su totalidad, ubicada esta última en el interior de la gran sala y ábside del edificio, no se hallaron restos óseos humanos durante la citada campaña debido a que fueron excavados en 1967-69. En cuanto a la distribución, teniendo en cuenta la edad de 
muerte estimada para los individuos, es el Área 1000 (parte superior de la Figura 5) la que presentó la mayor proporción de restos no-adultos (54\%), registrando el $87 \%$ y $90 \%$ de los individuos pertenecientes a los grupos 0-4 y 5-9 años respectivamente. En el propio Sector 1, el porcentaje de no-adultos se elevó hasta el $63 \%$, agrupando a cerca del $60 \%$ de los no-adultos de entre 0 y 9 años, siendo el único sector del yacimiento en el que fueron hallados individuos con una edad de muerte estimada inferior al primer año de vida $(\mathrm{N}=$ 18). El Sector 4, situado al sur del Sector 1, registró el segundo dato mayor de no-adultos por regiones, con un $48 \%$ de no-adultos y entre un 20 y $25 \%$ de los individuos del primer y segundo grupo de edad. Asimismo, fue posible observar como la proporción era considerablemente menor en las zonas cercanas al nártex (Área 4000, parte inferior de la Figura 5) con un porcentaje de individuos no-adultos inferior al $20 \%$ en la zona este.

En cuanto a la distribución en función del sexo de los individuos (Figura 6), en el Área 1000 se observó un número alto de indeterminaciones (64\%), correspondiéndose, mayoritariamente, con la elevada proporción de no-adultos, mientras que los individuos femeninos y masculinos se encontraron representados de forma prácticamente equitativa. Esta distribución homogénea parecía mantenerse constante en las distintas áreas a excepción del Área 3000, situada al oeste del edificio y que registró el doble de individuos masculinos (40\% - 6 casos- frente al 20\% -3 casos- de femeninos), así como en la sectorización del Área 4000, donde el Sector 3, localizado al noreste, registró una mayor frecuencia de individuos femeninos (53\% -9 casos- frente al 35\% -6 casos- de masculinos).

\subsection{Estudio paleodemográfico}

El cálculo de la tabla de vida proporcionó como resultado una estimación de la esperanza de vida al nacimiento de 25,55 años (Tabla 1). Dicho parámetro registró un ligero aumento con la edad, para llegar a un máximo

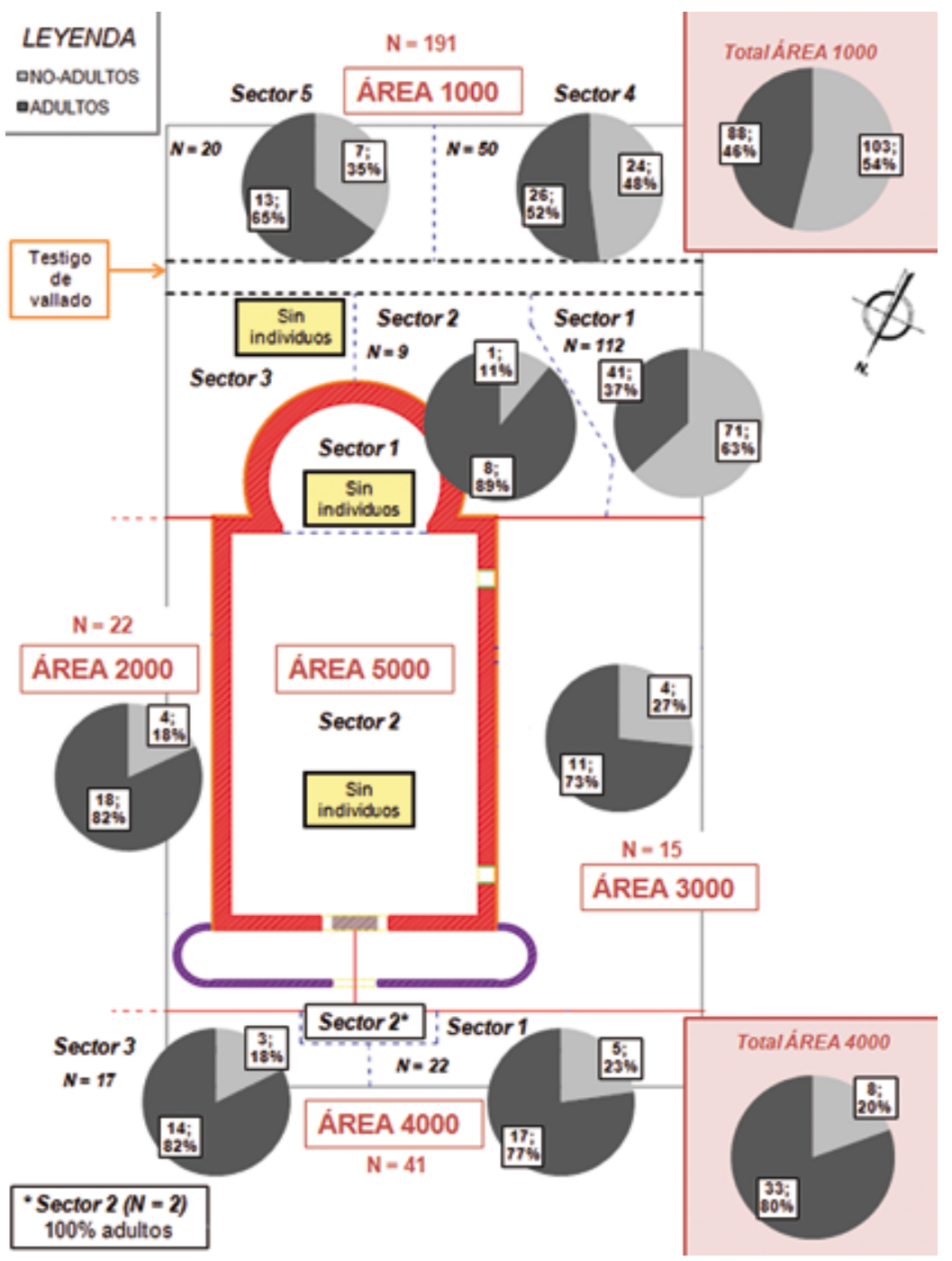

Fig. 5. Distribución de los individuos por edad en el espacio funerario / Age groups distribution in the cemetery 


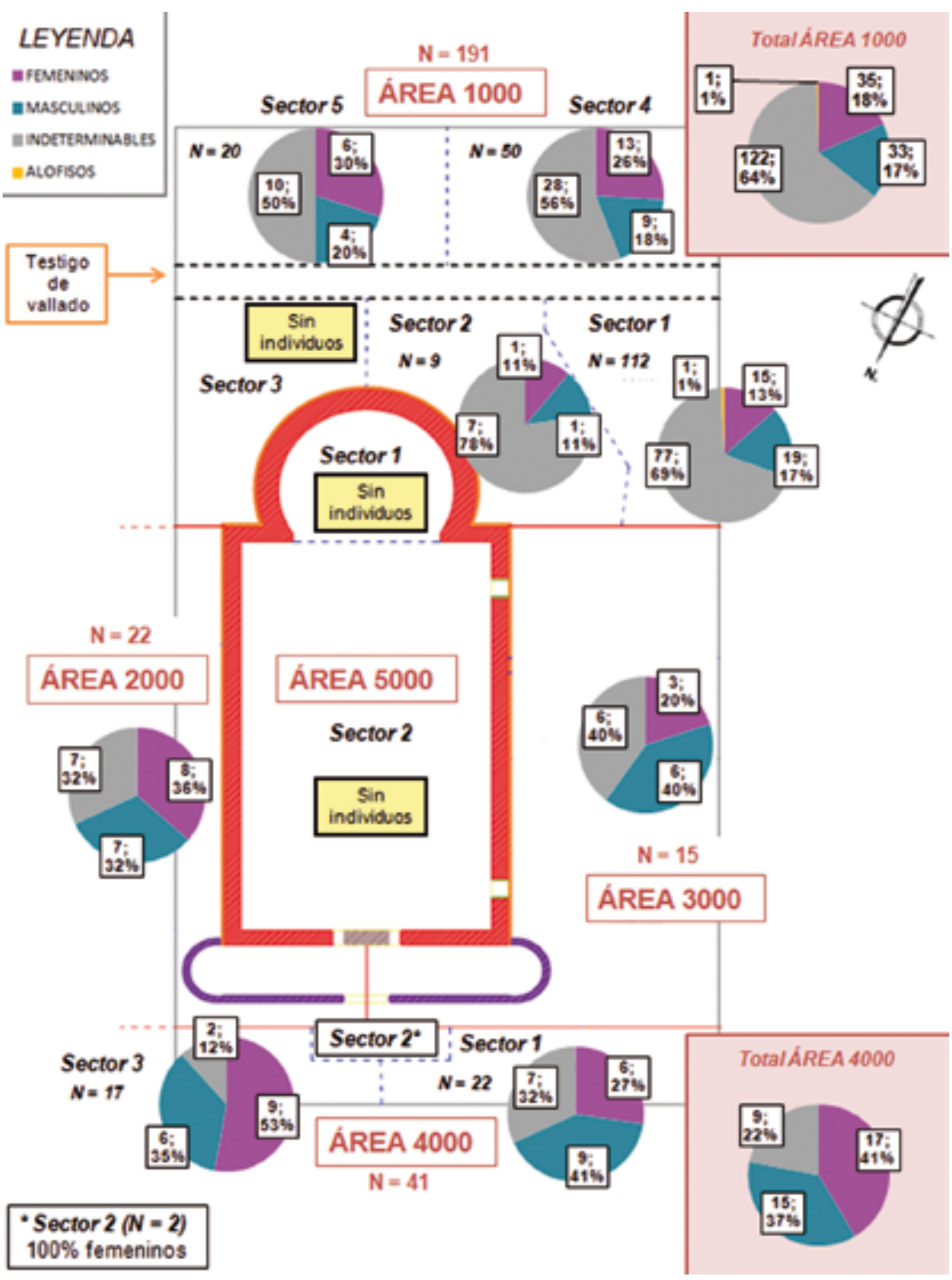

Fig. 6. Distribución de los individuos por sexo en el espacio funerario / Sex distribution in the cemetery.

\begin{tabular}{|c|c|c|c|c|c|c|c|}
\hline GRUPO & $D(x)$ & $d(x)$ & $I(x)$ & $q(x)$ & $L(x)$ & $T(x)$ & $e^{0}(x)$ \\
\hline 0 - 4 años & 65 & 24,16 & 100 & 0,24 & 439,59 & 2555,39 & 25,55 \\
\hline 5 - 9 años & 43 & 15,99 & 75,84 & 0,21 & 339,22 & 2115,80 & 27,90 \\
\hline 10 - 14 años & 8 & 2,97 & 59,85 & 0,05 & 291,82 & 1776,58 & 29,68 \\
\hline 15 - 19 años & 3 & 1,12 & 56,88 & 0,02 & 281,60 & 1484,76 & 26,10 \\
\hline Adulto Joven & 27 & 10,04 & 55,76 & 0,18 & 456,69 & 1203,16 & 21,58 \\
\hline Adulto & 36 & 13,38 & 45,72 & 0,29 & 429,37 & 746,47 & 16,33 \\
\hline Adulto Maduro & 62 & 23,05 & 32,34 & 0,71 & 270,63 & 317,10 & 9,80 \\
\hline Adulto Senil & 25 & 9,29 & 9,29 & 1,00 & 46,47 & 46,47 & 5,00 \\
\hline TOTAL & 269 & 100 & & & & & \\
\hline \multicolumn{8}{|c|}{$\begin{array}{l}\text { - D(x).-No de muertos: número de esqueletos en la muestra cuya edad de muerte estimada está dentro de los límites de la clase de edad. } \\
\text { - } d(x) \text {.-Porcentaje de muertos: porcentaje de los muertos de la población total incluido en la clase de edad. } \\
\text { - I(x).-Porcentaje de supervivientes: porcentaje de la población que continúa viva al principio de la clase de edad. } \\
\text { - } q(x) \text {.-Probabilidad de muerte: tasa de mortalidad de los individuos de cada grupo de edad. Cociente entre el porcentaje de muertos de ese grupo y el } \\
\text { número de supervivientes del mismo. } \\
\text { - L(x).-Número de años vividos: número de años vividos por todos los individuos en dicha clase. } \\
\text { - T(x).-Número de años por vivir: número de años por vivir por todos los individuos a partir de la clase de edad. } \\
\text { - e } e^{0}(x) \text {.-Esperanza de vida: media de edad que espera vivir un individuo de la clase de edad. }\end{array}$} \\
\hline
\end{tabular}

Tabla 1: Tabla de vida de la población de Marialba de la Ribera. / Life table of the Marialba de la Ribera population 


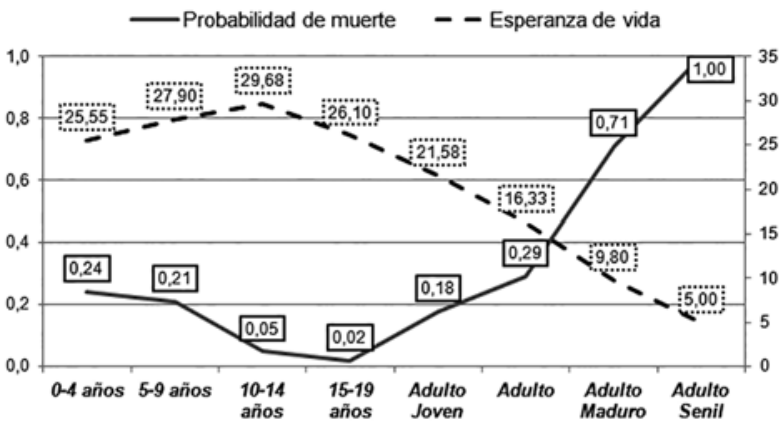

Fig. 7. Perfil de la probabilidad de muerte $(q(x))$ y la esperanza de vida $(e(x))$ de la colección. / Probability of death $(q(x))$ and life expectancy $(\mathrm{e}(\mathrm{x}))$ from the sample.

en los individuos con una edad de muerte estimada entre 10 y 14 años.

Los valores calculados relativos a la esperanza de vida se representan gráficamente en la Figura 7. Se puede observar el aumento de la esperanza de vida, con un máximo en el tercer grupo de edad (10-14 años) y un mínimo en el último (adultos seniles). Paralelamente, en cuanto a la probabilidad de muerte o cociente de mortalidad, este valor mostró una disminución progresiva hasta el grupo de edad 15-19 años, donde alcanzó su mínimo con un resultado de 0,02, debido a que sólo se recuperaron tres individuos correspondientes a este grupo de edad. A partir de dicho momento, el cociente de mortalidad aumenta a lo largo de los siguientes grupos.

También se realizó una comparación del cociente de mortalidad de la población de Marialba de la Ribera con los intervalos de confianza de las tablas-tipo (Figura 8). En el primer grupo de edad el cociente de mortalidad se situó por debajo del rango esperado, evidenciándose una subrepresentación de los individuos del grupo 0-4 años, mientras que en el siguiente grupo se localizó considerablemente por encima del límite propuesto, manifestando una marcada sobrerepresentación de los no-adultos de entre 5 y 9 años. Posteriormente, el cociente de mortalidad disminuyó hasta situarse dentro del intervalo para el grupo 10-14 años y ligeramente por debajo en el último grupo de edad (15-19 años), aunque con un dato muy próximo al propuesto como límite inferior.

Por último se calcularon los estimadores paleodemográficos propuestos por Masset y Bocquet, cuyos resultados se muestran en la Tabla 2 . Se puede observar que la esperanza de vida al nacimiento se estimó entre 13,59 y 16,60 años, un dato notablemente inferior al obtenido previamente mediante la tabla de vida. Por otro lado, el cociente de mortalidad para individuos con edades comprendidas entre 0 y 5 años calculado a través de esta metodología arrojó un dato de entre 0,51 y 0,59 , que es más del doble que el obtenido en la tabla de vida para este primer grupo de edad. Las tasas anuales de natalidad y mortalidad ofrecen valores muy similares, tanto en el supuesto de poblaciones estacionarias como contemplando crecimientos moderados. Finalmente, la tasa anual de fecundidad obtuvo un resultado de entre 0,2 y 0,21 , resultado que se traduciría en un número medio de siete hijos por mujer durante la totalidad de su etapa fértil.

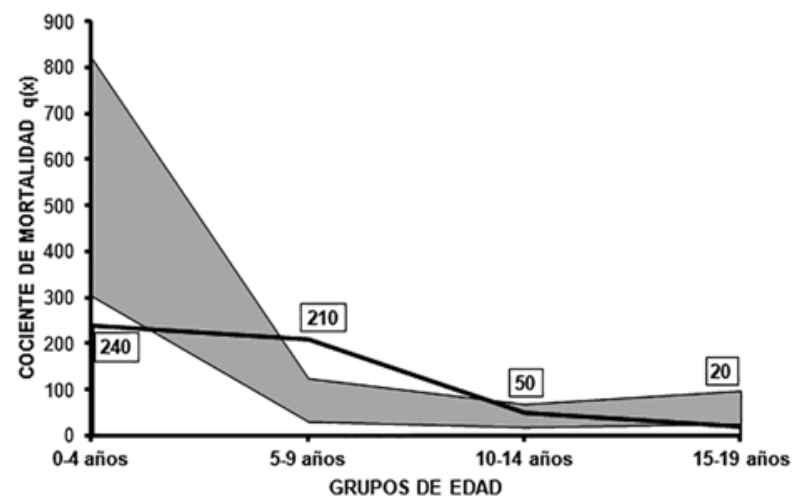

Fig. 8. Cociente de mortalidad calculado para la población de Marialba de la Ribera, comparado con los intervalos de confianza para poblaciones con una esperanza de vida entre 25 y 35 años (LEDERMANN, 1969). I Death probability in the Marialba de la Ribera population compared with the confidence intervals for population with life expectancies between 25 and 35 years (LEDERMANN, 1969).

\begin{tabular}{|c|c|c|c|c|c|c|}
\hline \multirow{3}{*}{$\begin{array}{l}\text { Índice de Ju- } \\
\text { ventud (I.J.) }\end{array}$} & \multirow{3}{*}{$\begin{array}{l}\text { Esperanza de } \\
\text { vida al naci- } \\
\text { miento }\left(e^{0}\right)\end{array}$} & \multirow{3}{*}{$\begin{array}{c}\text { Cociente de } \\
\text { mortalidad } \\
\text { [0-5 años] } \\
\left({ }_{5} q_{0}\right)\end{array}$} & \multicolumn{3}{|c|}{ Tasa anual de natalidad (n) y mortalidad (m) } & \multirow{3}{*}{$\begin{array}{c}\text { Tasa anual de } \\
\text { fecundidad para } \\
\text { mujeres entre } 15 \\
\text { y } 50 \text { años }\left({ }_{35} F_{15}\right)\end{array}$} \\
\hline & & & \multirow{2}{*}{$\begin{array}{c}\begin{array}{c}\text { Poblaciones } \\
\text { estacionarias }\end{array} \\
m=n\end{array}$} & \multicolumn{2}{|c|}{$\begin{array}{l}\text { Poblaciones en } \\
\text { crecimiento moderado }\end{array}$} & \\
\hline & & & & $n$ & $m$ & \\
\hline 0,34 & $13,59-16,60$ & $0,51-0,59$ & 0,06 & $0,05-0,06$ & $0,05-0,06$ & $0,20-0,21$ \\
\hline \multicolumn{7}{|c|}{$\begin{array}{l}- \text { I.J. }=D(5-14 \text { años }) / D(20-\infty \text { años }) \\
-e^{0}=70,721^{*} \log _{10} \sqrt{ }(1 / \text { I.J. }) \\
-{ }_{5} \mathrm{~g}_{0}=1,154^{*}\left(\log _{10}(200 * \text { I.J. })^{1 / 2}-1,014 \pm 0,041\right. \\
-m=n=\left(0,127^{*} \text { I.J. }\right)+0,016 \pm 0,002 \\
-n=\left(0,116^{*} \text { I.J. }\right)+0,018 \pm 0,003 \\
-m=\left(0,117^{*} \text { I.J. }\right)+0,013 \pm 0,003 \\
-{ }_{35} F_{15}=\left(-0,6601^{*}(\text { (I.J. })^{1 / 2}+(1,2419 \text { (I.J. })^{0,3}\right)-0,308 \pm 0,005\end{array}$} \\
\hline
\end{tabular}

Tabla 2: Estimadores paleodemográficos calculados para los individuos recuperados de Marialba de la Ribera (MASSET Y BOCQUET, 1977). / Paleodemographic estimators for Marialba de la Ribera archaological population (MASSET AND BOCQUET, 1977). 


\section{DISCUSIÓN}

La excelente preservación macroscópica de los restos óseos analizados y la baja tasa de reutilización del espacio funerario (1,14 individuos/tumba), han favorecido la obtención de resultados precisos en la caracterización antropológica de los individuos recuperados en el yacimiento. La tasa de reutilización condiciona notablemente la información que es posible obtener de los esqueletos, debido a que la acción antrópica llevada a cabo sobre los restos óseos ya esqueletizados, al recolocarlos desde su posición original a un depósito secundario generalmente más reducido, se va a traducir en peores resultados de preservación y consecuente pérdida de información en comparación con aquellas inhumaciones que hayan mantenido su posición primaria. La baja presión ejercida por las reutilizaciones en el cementerio de Marialba de la Ribera contrasta con lo observado en otras colecciones de similares características, destacándose el caso de Veranes (Gijón) con hasta 2,9 individuos/tumba (RASCÓN et al., 2011). En este sentido, es importante recalcar que el estudio llevado a cabo sobre la serie de Marialba de la Ribera, ha permitido estimar con fiabilidad la edad de muerte del 92\% de los individuos no-adultos y del $73 \%$ de los individuos adultos. Además, el uso de un amplio abanico de metodologías para la caracterización antropológica de los individuos también ha permitido determinar el sexo del $82 \%$ de los adultos. Estos excelentes resultados pueden atribuirse no sólo a la ya mencionada baja tasa de reutilización del cementerio, sino también a la participación de equipos de antropólogos desde los trabajos de campo. El conocimiento que posee el antropólogo de la anatomía humana permite realizar una documentación y recuperación de los restos mucho más precisa que en aquellos casos en que se prescinde de su participación. Además, posibilita la creación de un continuo de trabajo desde el campo al laboratorio, hecho constatado que aumenta notablemente la eficacia de los investigadores así como la validez de los posteriores estudios. Por todo ello y atendiendo a estos datos realmente excepcionales, es posible considerar el análisis paleodemográfico realizado sobre la población arqueológica recuperada como sólido y fiable.

En primer lugar, en cuanto a la estructura por grupos de edad obtenida para la población arqueológica, la proporción de no-adultos, con un dato del $44 \%$, se sitúa cercana al 50\% que cabría esperar en un cementerio procedente de épocas históricas, donde el número de individuos adultos y no-adultos debería ser equivalente (KRENZER, 2006). La distribución por grupos de edad en la etapa no-adulta muestra diferencias al comparar con el conjunto de otras poblaciones medievales del Antiguo Reino de León, como son los yacimientos de Saldaña (Palencia), Muriel de Zapardiel y San Benito (Valladolid) y Santo Tomé (Zamora). Esto podría atribuirse a que el grupo 5-9 años aparece sobrerrepresentado en la colección de Marialba de la Ribera, con un $16 \%$ respecto del total $(\mathrm{N}=43)$, mientras que en el conjunto de los yacimientos citados esta cifra tan sólo alcanza el 8\% (LÓPEZ, 2002). Sin embargo esta condición sí ha podido ser evidenciada en un contexto paleocristiano de otra región, el yacimiento de Veranes (Gijón), que incluso supera la cifra obtenida con un $21 \%$ de individuos en el grupo de 5-9 años (GONZÁLEZ et al., 2016). Igualmente, respecto a la determinación del sexo de los individuos, no ha sido posible apreciar una distribución diferencial en el conjunto ni en ninguna de las etapas del ciclo vital. Asimismo, el porcentaje de individuos clasificados como adultos indeterminables y alofisos ha resultado ser bajo (inferior al 20\%), lo que parece demostrar la inexistencia de mortalidad diferencial por sexos. Este resultado difiere de lo obtenido en el yacimiento de La Olmeda (Palencia), donde se documenta una acusada mortalidad femenina (CAMPILLO, 1995), aunque no parece resultar disonante con lo observado en otras poblaciones medievales de Castilla y León (LÓPEZ, 2002). No es posible establecer una comparación con el cementerio de Veranes (Gijón) ya que, aunque parece presentar mayor porcentaje de individuos masculinos, casi un tercio de sus individuos adultos han sido considerados como indeterminables (GONZÁLEZ et al., 2016).

Cuando se analiza la distribución de los individuos a lo largo del espacio funerario, por un lado teniendo en cuenta el grupo de edad al que pertenecen, es posible determinar que en el cementerio de Marialba de la Ribera parece existir una zona preferente para el entierro de los perinatales. Esto es debido a que sólo se han recuperado individuos cuya edad de muerte fue estimada en menos de un año en el Sector 1 del Área 1000. Dicho sector junto con el Sector 4 de la misma área, localizados en la región sur excavada, parecen ser los lugares de enterramiento preferencial para los individuos de los dos primeros grupos de edad (0-9 años), ya que el $87-90 \%$ de los mismos han sido recuperados en esta zona del yacimiento y, en particular, en la última fase de enterramientos (ss. XII-XIII). Además, es posible observar lo que podría considerarse como un gradiente de distribución en relación con la orientación geográfica, en el que los individuos no-adultos aparecen menos representados en las distintas áreas y sectores más alejados de la zona sur-suroeste, mostrando proporciones incluso superiores al $60 \%$ en dicha región e inferiores al $20 \%$ en el noreste. La existencia de particularidades en la distribución de los no-adultos en este cementerio ya fue evidenciada en el análisis preliminar de los resultados obtenidos en los trabajos de campo (GONZÁLEZ et al., 2013), determinándose el Área 1000 como el "limbo de los niños" (GONZÁLEZ et al., 2006) y proponiéndose la existencia de un gradiente, paralelo al eje mayor de la edificación, relativo a la frecuencia de aparición de restos no-adultos. El avance de la investigación ha permitido precisar en esta ocasión que los perinatales tan sólo se localizan en un sector de dicha área (Sector 1), y que no sólo éstos, sino todos los individuos de entre 0 y 9 años, parecen poseer un lugar preferencial de enterramiento en la región sur-suroeste. Igualmente, 
ha sido posible concretar que el gradiente no parece responder tanto a la estructura de la edificación sino a la orientación geográfica (norte-sur). Por todo ello, esta distribución parece indicar que el yacimiento de Marialba de la Ribera supone otro ejemplo de uso diferencial del espacio funerario en función del grupo de edad, hecho que ya ha sido documentado en otros contextos cristianos en España (GÓMEZ Y GONZÁLEZ, 2009; IGLESIAS Y GONZÁLEZ, 2009; PEÑA, 2013) y en otras regiones geográficas. En relación con este hallazgo se encontrarían las conocidas como "necrópolis de bebés", ampliamente documentadas en Francia (GÉLIS, 2006; DELATTRE, 2008; TZORTZIS Y SÉGUY, 2008). En ellas es posible observar un tratamiento diferencial en las inhumaciones de los individuos de menor edad, representando lo que ha sido descrito como bautismos de "urgencia" o santuarios de resurrección momentánea (sanctuaire à répit). Aunque la falta de documentación escrita dificulta su correcta interpretación, parece existir consenso en considerar que aquellos niños que fallecían antes de su confirmación (en torno a los 7 u 8 años de edad) no podían ser enterrados en similares condiciones a los adultos y, por tanto, disponían de un lugar particular en el cementerio, denominado como "la parcela de los niños" (SÉGUY, 1997; SÉGUY, 2010). Por otro lado, respecto de la distribución de individuos atendiendo al sexo, los únicos sectores en los que se encuentran desequilibrios evidentes en las proporciones entre sexos presentan un número escaso de individuos; por tanto, la importancia de este hecho es limitada, teniendo en cuenta la inexistencia de diferencias en el total del cementerio así como en las áreas y sectores con mayor número de enterramientos.

En cuanto al estudio paleodemográfico cabe destacar, por un lado, que el resultado previamente mencionado acerca de la inexistencia de mortalidad diferencial por sexos justifica el cálculo de una tabla de vida conjunta para toda la colección. De este modo se evita la problemática de realizar tablas para sexos separados cuando existen números de efectivos limitados y elevadas proporciones de indeterminaciones atribuibles a los individuos no-adultos. Así, el cálculo de la tabla de vida propuesta por ÁCSADI Y NEMESKÉRI (1970) proporciona una esperanza de vida al nacimiento para la población arqueológica de Marialba de la Ribera de 25,55 años. Este resultado es muy similar al obtenido en el análisis de las colecciones medievales del antiguo Reino de León (LÓPEZ, 2002), en el que se ha establecido una media en la esperanza de vida al nacimiento que se situaría en los 26,20 años, así como en el yacimiento de Veranes (Gijón), con una cifra de 26,31 años (GONZÁLEZ et al., 2016). El aumento de la esperanza de vida en los primeros grupos de edad, alcanzando el máximo en el grupo 10-14, es consecuencia última de la recuperación llevada a cabo sobre los individuos no-adultos, entre los que son muy frecuentes los asignados al grupo 5-9 años ( $N=43)$, en comparación con los grupos adyacentes $(0-4, N=65 ; 10-14, N=8)$. En un supuesto de mortalidad natural, lo esperable sería obtener el va- lor más alto en la segunda categoría de edad, al haber superado los individuos que constituyen dicho grupo la etapa previa, que es el periodo crítico en cuanto a la probabilidad de muerte. Sin embargo, en el cementerio de Marialba de la Ribera este dato máximo se sitúa en el tercer grupo de edad (10-14 años), y puede verse reflejado del mismo modo en el leve descenso que experimenta la probabilidad de muerte para el segundo grupo respecto del primero, relacionándose nuevamente con la alta representación de los individuos de 5-9 años y la subrepresentación del grupo 0-4 años. Esta circunstancia guarda relación con que la esperanza de vida al nacimiento sea un parámetro muy sensible al número de individuos recuperados de los primeros grupos de edad. Por tanto, el perfil obtenido para la esperanza de vida no concuerda, en el caso de los individuos no-adultos, ni con lo esperado en poblaciones arqueológicas ni con los yacimientos afines con los que ha sido comparado, debido a que en los mismos la mayor esperanza de vida se sitúa en el grupo 0-4 años (LÓPEZ, 2002; GONZÁLEZ et al., 2016).

Por otro lado, respecto del gráfico de la evolución de la probabilidad de muerte a través de las diferentes categorías de edad, se puede observar como la población del yacimiento presenta grandes similitudes con el perfil de mortalidad típico de las poblaciones arqueológicas. Dicho perfil debe exhibir una característica forma de "U" (JACKES, 2011), debido a que las menores frecuencias se sitúan en las etapas intermedias, tal y como le corresponde a los individuos no-adultos de entre $10 \mathrm{y}$ 19 años y a los adultos jóvenes, mientras que la mayor mortalidad puede observarse en los primeros años de vida y en las etapas avanzadas del ciclo vital (GONZÁLEZ, 2008). En el caso de la población arqueológica de Marialba de la Ribera tan sólo parecen desviarse de lo esperado los individuos del grupo 0-4 años, ya que su probabilidad de muerte es proporcionalmente baja, reflejando una posible subrepresentación de los individuos de dicho grupo. El perfil obtenido concuerda con el conjunto de colecciones castellano leonesas (CAMPILLO, 1995; LÓPEZ, 2002).

Posteriormente, a fin de evaluar de forma más específica el comportamiento de los individuos no-adultos de la colección, se ha recurrido a las tablas-tipo de mortalidad para comparar el cociente de mortalidad calculado con el esperado para poblaciones con $e^{0}$ entre 25 y 35 años. Los resultados evidencian el desequilibrio en los dos primeros grupos de edad, observándose subrepresentación del grupo 0-4 años así como acusada sobrerepresentación en el grupo 5-9 años, ya detectadas en otros análisis. Los resultados obtenidos para el resto de no-adultos pueden considerarse que son concordantes con los datos esperados de mortalidad pretransicional.

Finalmente, en lo que respecta al cálculo de los estimadores paleodemográficos propuestos por MASSET Y BOCQUET (1977), la esperanza de vida al nacimiento obtenida, con un dato que oscila entre los 13,59 y los 16,60 años, resulta incompatible con una dinámica po- 
blacional normal ya que a esa edad apenas se ha alcanzado la etapa reproductora del ciclo vital humano. Este resultado sólo puede ser explicado debido, de nuevo, a la elevada proporción de individuos del grupo 5-9 años, que forman parte del cálculo del Índice de Juventud, por lo que se obtiene una cifra alta del mismo $(0,34)$ que condiciona el resultado de los estimadores. Igualmente, es posible observar como el cociente de mortalidad estimado para los individuos de la primera categoría de edad, con un valor de más del doble del obtenido a través de la tabla de vida, refleja que el número de individuos recuperados para ese grupo de edad es escaso. La similitud entre los valores estimados de mortalidad y natalidad parecen indicar que la población estudiada se comportaría como una población estable o estacionaria; contrariamente, los siete hijos por mujer estimados a partir de la tasa anual de fecundidad podrían indicar un fuerte crecimiento natural de la población. En la literatura paleodemográfica se encuentran numerosas referencias a poblaciones arqueológicas con valores de descendencia de entre siete y ocho hijos por mujer (ÁCSADI Y NEMESKÉRI, 1970), pero la realidad es que un patrón de crecimiento de este estilo sería incompatible con un modelo sostenible en el periodo histórico estudiado (BOCQUET, 1979). En la población de Marialba de la Ribera, el exceso de representantes del grupo 5-9 años condiciona el cálculo de dichos parámetros.

En definitiva, sería importante destacar que el yacimiento de Marialba de la Ribera en la actualidad, a pesar de su potencialidad tanto arqueológica como antropológica en el contexto paleocristiano del norte peninsular presenta, de cara a un análisis paleodemográfico, las limitaciones propias derivadas de una excavación que no ha sido exhaustiva ni completa. Recientes aproximaciones parecen indicar que el cementerio se extiende hacia el oeste y, ya que los resultados de este estudio han constatado que la zona preferencial de enterramiento de los individuos de 0-9 años se encuentra en la región sur-suroeste, cabría esperar que no se haya recuperado a día de hoy la totalidad de los individuos muertos en las primeras etapas del ciclo vital. Este hecho estaría generando un sesgo y quizá permitiría explicar por completo la subrepresentación entre los 0-4 años y la sobrerepresentación entre los 5-9 años halladas en el estudio. Esta circunstancia es realmente importante, ya que una frecuencia por encima de lo esperado podría ser interpretada como una crisis de mortalidad (p. e., epidemias), cuando la solución muy posiblemente se encuentre en el sesgo introducido en la representación de la población de estudio a consecuencia de la excavación parcial del cementerio (PÉREZ, 2010). Del mismo modo, los restos óseos humanos que fueron recuperados por los equipos alemanes en las cerca de cien tumbas halladas, no han podido ser unificados y reanalizados con el material utilizado en la presente investigación. Se estima que su número mínimo de individuos será similar o superior al número de enterramientos y, por tanto, va a suponer una proporción considerable del total de la colección. El des- conocimiento de la composición por grupos de edad y sexo de esta gran cantidad de individuos dificulta la interpretación de los resultados presentados en este trabajo. Sólo incorporando al estudio antropológico la totalidad de los individuos preservados en el yacimiento será posible conocer, en profundidad y sin sesgos, la estructura y dinámica de la población de los antiguos habitantes de Marialba de la Ribera.

\section{CONCLUSIONES}

Los restos óseos estudiados en la colección de Marialba de la Ribera corresponden a 269 individuos, distribuidos en 237 enterramientos. La preservación de la colección puede definirse como muy favorable para el estudio antropológico. Este hecho, unido a la diversidad de métodos empleados en la caracterización antropológica individual, ha permitido que el 92\% de los individuos no-adultos y el 73\% de los adultos hayan sido clasificados en un grupo de edad concreto. Igualmente, respecto de la determinación del sexo, el $82 \%$ de los individuos adultos ha podido ser adjudicado a las categorías "femenino" y "masculino". Los análisis han permitido concluir que no existe mortalidad diferencial por sexos en la etapa adulta y que la mortalidad registrada por categorías de edad, a partir del grupo 10-14 años, se ajusta al esperado en un supuesto natural o pretransicional. Las desviaciones dentro de este supuesto natural, evidenciadas en las distintas aproximaciones realizadas para el análisis paleodemográfico de la población arqueológica de Marialba de la Ribera, ponen de manifiesto la subrepresentación de individuos del grupo 0-4 años y la sobrerepresentación en el 5-9 años. El análisis en profundidad de la distribución de individuos en el cementerio ha permitido identificar en este trabajo la existencia de un uso diferencial del espacio funerario. De este modo, y debido a la excavación no exhaustiva del cementerio, cabría esperar que los individuos de menor edad no hubieran sido recuperados en su totalidad. Las áreas 1000 y 4000, localizadas en la región sur-suroeste del yacimiento, previsiblemente contendrán dichos no-adultos, motivo por el cual parece recomendable dar continuidad a las excavaciones en este conjunto arqueológico.

\section{AGRADECIMIENTOS}

Este trabajo ha sido realizado vinculado a los Proyectos de Investigación "De Conventus Asturum a Asturorum Regnum. El territorio de la ciuitas Legione y el asentamiento de Marialba de la Ribera (León) entre época tardoantigua y medieval" (MCINN-12HAR2011-23106), el "Proyecto de recuperación, protección y puesta en valor del monumento de Marialba", promovido por la Fundación del Patrimonio Histórico de Castilla y León, y "Formas de ocupación y organización del espacio en el norte peninsular: el territorio astur entre época antigua y medieval a través del registro arqueoló- 
gico y paleoambiental" (HAR2016-78036-P), financiado por el Ministerio de Economía y Competitividad.

Los autores agradecen especialmente al Dr. Theodor Hauschild del Deutsches Archäologisches Institut (DAl) de Madrid, el apoyo prestado durante el desarrollo de la investigación.

\section{BIBLIOGRAFÍA}

ACSÁDI, G.Y. \& NEMESKÉRI, J.

1970 History of human life span and mortality. Akadémiai Kiadó. Budapest.

\section{BOCQUET, J.P.}

1979 Une approche de la fécondité des populations inhumées. Bulletin et Mémoires de la Société d'Anthropologie de Paris 6(3), 261-268.

B.O.E.

1979 Decreto 89/2000, de 27 de abril, por el que se adecua la declaración de bien de interés cultural de la iglesia Martirial, en Marialba de la Ribera, municipio de Villaturiel (León), como zona arqueológica, delimitando el área afectada por su declaración. Boletín Oficial del Estado 178 (26 de julio de 2000), 26707-26708.

BROWN, M.A.

1983 Grave orientation: a further view. The Archaeological Journal 140, 322-328.

BRUZEK, J.

2002 A method for visual determination of sex, using the human hip bone. American Journal of Physical Anthropology 117(2), 157-168.

BRUZEK, J. \& MURAIL, P.

2006 Methodology and reliability of sex determination from the skeleton, en SCHMITT, E., CUNHA, E. \& PINHEIRO, J. Forensic Anthropology and Medicine. Complementary sciences from recovery to cause of death. 225242. Humana Press. Totowa, New Jersey.

BRUZEK, J., CASTEX, D. et MAJÓ, T.

1996 Évaluation des caractères morphologiques de la face sacro-pelvienne de l'os coxal. Proposition d'une nouvelle méthode de diagnose sexuelle. Bulletin et Mémoires de la Société d'Anthropologie de Paris 8(3-4), 491-502.

BUIKSTRA, J.E.

1976 Hopewell in the Lower Illinois Valley: A regional study of human biological variability and prehistoric mortuary behavior. Northwestern University Archaeological Program, Scientific Papers, 2. Evanston, Illinois.

\section{CAMPILLO, D.}

1995 Mortalidad y esperanza de vida en la Península Ibérica, desde la Prehistoria a la Edad Media, en FÁBREGAS, R., PÉREZ, F. y FERNÁNDEZ, C. Arqueoloxía da Morte na Península Ibérica desde as Orixes ata o Medievo. 317-340. Excmo. Concello, Biblioteca Arqueohistórica Limiá, Serie Cursos e Congresos 3. Xinzo de Limia.
CARRO, J

1970 Estudio anatomoantropológico de los restos humanos del templo de Marialba, en Legio VII Gemina. 522-548. León.

\section{DELATTRE, V.}

2008 Les sépultures de nouveau-nés au Moyen-Âge : l'hypothèse d'un sanctuaire à répit précoce a Blandy-lesTours (France, Seine-et-Marne), en GUSI, F., MURIEL, S. et OLARIA, C.R. Nasciturus, infans, puerulus vobis mater terra: La muerte en la infancia. 183-210. Diputació de Castelló, Servei d'Investigacions Arqueològiques i Prehistòriques. Castellón.

\section{DEUTSCH, D., TAM, O. \& STACK, M.V.}

1985 Postnatal changes in size, morphology and weight of developing postnatal deciduous anterior teeth. Growth 49, 202-217.

FAZEKAS, I.G. \& KÓSA, F.

1978 Forensic fetal osteology. Akadémiai Kiadó. Budapest.

FEREMBACH, D., SCHWIDETZKY, I. \& STLOUKAL, M.

1980 Recommendations for age and sex diagnoses of skeletons. Journal of Human Evolution 9, 517-549.

GÉLIS, J.

2006 Les enfants des limbes. Mort-nés et parents dans l'Europe chrétienne. Audibert. Paris.

GÓMEZ, A. y GONZÁLEZ, A.

2009 El "limbo de los niños" de la ermita de San Julián y Santa Basilișa (Zalduondo, Álava), en POLO, M. y GARCÍA-PRÓSPER, E. Investigaciones histórico-médicas sobre salud y enfermedad en el pasado. Actas del IX Congreso Nacional de Paleopatología. 733-736. Grupo Paleolab, Sociedad Española de Paleopatología. Valencia.

\section{GÓMEZ-MORENO, M.}

1925 Catálogo monumental de España. Provincia de León (1906-1908). Ministerio de Instrucción Pública y Bellas Artes. Madrid.

\section{GONZÁLEZ, A.}

1999 Infancia y adolescencia en la Murcia musulmana. Estudio de restos óseos. Tesis Doctoral. Universidad Autónoma de Madrid. Madrid.

2008 Mitos y realidades en torno a la excavación, el tratamiento y el estudio de los restos arqueológicos no-adultos, en GUSI, F., MURIEL, S. y OLARIA, C.R. Nasciturus, infans, puerulus vobis mater terra: La muerte en la infancia. 57-76. Diputació de Castelló, Servei d'Investigacions Arqueològiques i Prehistòriques. Castellón.

GONZÁLEZ, A., RASCÓN, J., CAMBRA-MOO, O., PIMENTEL, G. y CAMPO, M.

2016 Estudio poblacional del cementerio medieval de Veranes (Gijón, Asturias): estado de la cuestión, en QUIRÓS, J.A. (Dir.). Demografía, paleopatologías y desigualdad social en el noroeste peninsular en época medieval. 83-95. Documentos de Arqueología Medieval 10. Servicio Editorial de la Universidad del País Vasco. Bilbao. 
GONZÁLEZ, A., GÓMEZ, A. y ETXEBERRIA, F.

2006 Contribución de la osteoarqueología al debate sobre la existencia o inexistencia del limbo. Boletín de la Asociación Española de Paleopatología 44, 9-10.

GONZÁLEZ, A., VEGA, R., ELVIRA, A., SAN ROMÁN, F. y CAMBRA-MOO, O.

2013 Distribución espacial de los enterramientos infantiles en la necrópolis de la basílica paleocristiana de $\mathrm{Ma}$ rialba de la Ribera (Villaturiel, León), en MALGOSA, A. ISIDRO, A., IBÁÑEZ-GIMENO, P. y PRATS-MUÑOZ, G. Vetera Corpora Morbo Afflicta. Actas del XI Congreso Nacional de Paleopatología. 305-310. Universitat Autònoma de Barcelona. Barcelona.

GUTIÉRREZ, J.A.

2015 La temprana Edad Media en tierras de León (siglos V-VIII), en ArqueoLeón II. Historia de León a través de la Arqueología: actas, Museo de León, noviembre 2013/ marzo 2014. 157-176. Junta de Castilla y León, Diputación de León. León.

GUTIÉRREZ, J.A. y BENÉITEZ, C.

1996 Los tiempos oscuros: la transición a la Edad Media en tierras leonesas, en ArqueoLeón. Historia de León a través de la Arqueología: ciclo de conferencias, León 1993-1994. 107-122. Junta de Castilla y León, Consejería de Educación y Cultura. Valladolid.

GUTIÉRREZ, J.A., CAMPOMANES, E., MIGUEL, F., BENÉITEZ, C., MARTÍN, P., MUÑOZ, F.A. y SAN ROMÁN, F.

2010 Legio (León) en época visigoda: la ciudad y su territorio, en GARCÍA, A., IZQUIERDO, R., OLMO, L. \& PERIS, D. Espacios urbanos en el Occidente Mediterráneo (s. VI-VIII). 131-136. Toletum Visigodo. Toledo.

HAUSCHILD, T.

1968 La iglesia martirial de Marialba (León). Boletín de la Real Academia de la Historia 163(2), 243-249.

HOPPA, R.D. \& VAUPEL, J.W.

2002 Paleodemography. Age distributions from skeletal samples. Cambridge University Press. Cambridge.

IGLESIAS, J. y GONZÁLEZ, A.

2009 El "limbo de los niños" de la iglesia de San Juan Bautista. Plaza de Ramales (Madrid), en POLO, M. y GARCÍA-PRÓSPER, E. Investigaciones histórico-médicas sobre salud y enfermedad en el pasado. Actas del IX Congreso Nacional de Paleopatología. 729-731. Grupo Paleolab, Sociedad Española de Paleopatología. Valencia.

IRURITA, J., ALEMÁN, I., VICIANO, J., DE LUCA, S. \& BOTELLA, M.C.

2014 Evaluation of the maximum length of deciduous teeth for estimation of the age of infants and young children: proposal of new regression formulas. International Journal of Legal Medicine 128(2), 345-352.

JACKES, M.

2011 Representativeness and bias in archaeological samples, en AGARWAL S.C. \& GLENCROSS, B.A. Social Bioarchaeology. 107-146. Blackwell Publishing. Chichester.
KRENZER, U.

2006 Compendio de métodos antropológicos forenses para la reconstrucción del perfil osteo-biológico. Tomo IV: Estimación de la edad osteológica en subadultos. Centro de Análisis Forense y Ciencias Aplicadas (CAFCA). Guatemala.

\section{LEDERMANN, S.}

1969 Nouvelles tables-types de mortalité. Presses Universitaires de France. Institut National d'Etudes Démographiques. Paris.

LEWIS M.E.

2007 The Bioarchaeology of children. Perspectives from Biological and Forensic Anthropology. Cambridge University Press. Cambridge.

LIVERSIDGE, H.M., DEAN, M.C. \& MOLLESON, T.I.

1993 Increasing human tooth length between birth and 5.4 years. American Journal of Physical Anthropology 90(3), 307-313.

\section{LÓPEZ, B.}

2002 Los pobladores del Antiguo Reino de León: Antropometría, Paleodemografía, Paleopatología. Universidad de León. León.

\section{MASSET, C. et BOCQUET, J.P.}

1977 Estimateurs en paléodémographie. L'Homme 17(4), 65-90.

\section{MILES, A.E.W.}

1963 The dentition in the assessment of individual age in skeletal material, en BROTHWELL, D. Dental Anthropology. 191-209. Pergamon Press. New York.

MURAIL, P., BRUZEK, J., HOUËT F. \& CUNHA, E.

2005 DSP: a tool for probabilistic sex diagnosis using worldwide variability in hip-bone measurements. Bulletins et Mémoires de la Société d'Anthropologie de Paris 17(34), 167-176.

PEÑA, V.

2013 Infancia y espacio funerario: el cementerio parroquial medieval y moderno de San Andrés en Madrid, en MALGOSA, A., ISIDRO, A., IBÁÑEZ-GIMENO, P. y PRATS-MUÑOZ, G. Vetera Corpora Morbo Afflicta. Actas del XI Congreso Nacional de Paleopatología. 507526. Universitat Autònoma de Barcelona. Barcelona.

\section{PÉREZ, E.}

2010 La place de l'enfant dans l'espace funéraire du haut Moyen Âge (Vle-Xe siècle). Cahiers d'Histoire 29(2), 119-136.

\section{PHENICE, T.W}

1969 A newly developed visual method of sexing the os pubis. American Journal of Physical Anthropology 30(2), 297-302. 
RASCÓN, J., CAMBRA-MOO, O., PIMENTEL, G., GONZÁLEZ, A. y CAMPO, M.

2011 Influencia del estado de preservación de los restos óseos en el diagnóstico paleopatológico, en GONZÁLEZ, A., CAMBRA-MOO, O., RASCÓN, J., CAMPO, M., ROBLEDO, M., LABAJO, E. y SÁNCHEZ, J.A. Paleopatología: ciencia multidisciplinar. Actas del X Congreso Nacional de Paleopatología. 45-59. Sociedad Española de Paleopatología, Universidad Autónoma de Madrid, Universidad Complutense de Madrid. Madrid.

\section{RUIZ, G. y CHAPA, M.T.}

1990 La arqueología de la muerte: perspectivas teórico-metodológicas, en BURILLO, F. Necrópolis celtibéricas: /I Simposio sobre los celtíberos [Daroca (Zaragoza), 28 al 30 de abril de 1988]. 357-374. Institución Fernando el Católico. Zaragoza.

SÁNCHEZ-MEJORADA, G., GÓMEZ-VALDÉS, J., HERRERA, P., VELEMISKY, P. y BRUZEK, J.

2011 Valoración del método de Diagnóstico Sexual Probabilístico (DSP) en una colección osteológica mexicana. Estudios de Antropología Biológica 15, 359-374.

SCHEUER, L. \& BLACK, S.

2000 Developmental juvenile osteology. Academic Press, Elsevier. London.
SÉGUY, I.

1997 Aspects religieux et profanes dans le traitement funéraire réservé au nouveau-né au Moyen Âge et à l'époque moderne, en BUCHET, L. L'enfant, son corps, son histoire. Actes des Septièmes Journées Anthropologiques de Valbonne, 1-3 juin 1994. 97-113. Éditions APDCA. Antibes.

2010 La muerte de los pequeñitos: entre el dogma y las creencias populares. Francia, de finales de la Antigüedad a la Época Moderna. Trace 58, 29-39.

TZORTZIS, S. et SÉGUY, I.

2008 Pratiques funéraires en lien avec les décès des nouveaux nés. À propos d'un cas dauphinois durant l'Époque moderne : la chapelle Saint-Jean à L'Argentière-La-Bessée (Hautes-Alpes). Socio-anthropologie 22, 75-92.

\section{UBELAKER, D.H.}

1978 Human skeletal remains: Excavation, Analysis, Interpretation. Taraxacum. Washington.

WALDRON, T.

1994 Counting the dead: The epidemiology of skeletal populations. Wiley. Chichester. 
